Methods We retrospectively analyzed patients diagnosed with 2009 FIGO stage IB1-IIA2 cervical cancer who underwent primary radical hysterectomy between 2007 and 2019. Using an artificial intelligence-based tool, the skeletal muscle area $\left(\mathrm{cm}^{2}\right)$ at the third lumbar vertebra (L3) and the skeletal muscle volume $\left(\mathrm{cm}^{3}\right)$ at the waist level were measured from pre-treatment CT scans. These were converted to the L3 and volumetric skeletal muscle indices (SMIs) by normalization. We defined L3 and volumetric sarcopenia using $39.0 \mathrm{~cm}^{2} / \mathrm{m}^{2}$ and the first quartile value, respectively. Patients' survival outcomes were compared according to the presence of sarcopenia.

Results A total of 306 patients were included. Between the L3 sarcopenia and non-sarcopenia groups, no differences in progression-free survival (PFS) and overall survival (OS) were observed. In contrast, the volumetric sarcopenia $(n=76)$ showed significantly worse PFS $(\mathrm{P}=0.039)$ and $\mathrm{OS}(\mathrm{P}=0.031)$ than did the volumetric non-sarcopenia group $(n=230)$. In multivariate analyses, volumetric sarcopenia was identified as a poor prognostic factor for PFS (aHR, 1.872; 95\% CI, 1.026-3.415; $\mathrm{P}=0.041$ ) and $\mathrm{OS}$ (aHR, 3.172; 95\% CI, $1.058-9.512 ; \mathrm{P}=0.039)$. Regarding changes in body composition, initial volumetric sarcopenia with total fat gain during primary treatment was associated with worse PFS (aHR, 3.015; 95\% CI, 1.314-6.919; $\mathrm{P}=0.009)$, but not $\mathrm{OS}$ $(\mathrm{P}=0.070)$.

Conclusions Volumetric sarcopenia increased the recurrence and mortality rates in patients with early cervical cancer. Patients with initial volumetric sarcopenia and total fat gain during primary treatment were at a high risk of disease recurrence.

\section{EPV078/\#473 EFFECTS OF PREOPERATIVE CERVICAL CONIZATION ON SURVIVAL OUTCOMES IN PATIENTS WITH EARLY-STAGE CERVICAL CANCER WHO UNDERGO PRIMARY RADICAL HYSTERECTOMY: A PROPENSITY SCORE MATCHING STUDY}

SI Kim*, HS Kim, HH Chung, J-W Kim, NH Park, Y-S Song, M Lee. Seoul National University College of Medicine, Department of Obstetrics and Gynecology, Seoul, Korea, Republic of

\subsection{6/ijgc-2021-IGCS.146}

Objectives To ascertain whether preoperative cervical conization decreases disease recurrence and mortality rates in patients with early cervical cancer who undergo radical hysterectomy (RH).

Methods We retrospectively identified 2014 FIGO stage IB cervical cancer who received primary $\mathrm{RH}$ by either minimally invasive surgery (MIS) or open surgery between 2005 and 2020. To adjust for confounders, we conducted a 1:2 propensity score matching for stage, histology, cervical mass size, and surgical approach. Then, survival outcomes were compared between the matched conization and non-conization groups.

Results A total of 429 patients were included: 96 (22.4\%) received preoperative conization. Overall, the conization group had significantly less cervical mass size (median, 24.0 vs. $30.0 \mathrm{~mm} ; \mathrm{P}=0.020)$ and lower incidence rates of parametrial invasion ( $4.2 \%$ vs. $15.0 \% ; \mathrm{P}=0.005)$, compared to the non-conization group. The conization group had a trend towards MIS RH (54.2\% vs. 43.2\%; $\mathrm{P}=0.058)$. After matching, the conization group showed significantly better progression-free survival (PFS) than the non-conization group $(\mathrm{n}=192) \quad(3$-year: $96.8 \%$ vs. $86.5 \% ; \mathrm{P}=0.011)$, but no difference in overall survival (OS). Excluding 15 patients who had parametrial invasion, lymph node metastasis, and both, we conducted another matching process and also found that the conization group had significantly better PFS (3-year: $86.1 \%$ vs. $98.8 \% ; \mathrm{P}=0.008$ ), but the similar OS. Consistent results were also observed in the subgroup of MIS RH $(n=150)$.

Conclusions Despite the retrospective design, our matched cohort study suggests that preoperative conization might be preferable for the surgical treatment of FIGO stage IB cervical cancer, especially for those who are planning to undergo MIS RH.

\section{EPV079/\#495 ACCURACY OF MAGNETIC RESONANCE IMAGING FOR PREOPERATIVE PREDICTION OF PATHOLOGIC TUMOR SIZE AND THE NEED OF ADJUVANT RADIOTHERAPY IN EARLY-STAGE CERVICAL CANCER}

D Kim*, U Kim, SJ Park, M Lee, HS Kim, HH Chung, J-W Kim, NH Park, Y-S Song. Seoul National University Hospital, Gynecology, Seoul, Korea, Republic of

\subsection{6/ijgc-2021-IGCS. 147}

Objectives To evaluate the accuracy of magnetic resonance imaging (MRI) for preoperative prediction of pathologic tumor size and the need of adjuvant radiotherapy in early-stage cervical cancer.

Methods We included patients with the following criteria: stage IB1-IIA2 cervical cancer; no diagnostic conization; visible tumors on MRI; no risk factors such as lympho-vascular space invasion, parametrial invasion, positive resection margin and lymph node metastasis. Adjuvant radiotherapy was applied in those with tumor size $\geq 4 \mathrm{~cm}$ and stromal invasion $>1 / 2$.

Results We collected data of 102 patients with the criteria mentioned above between 2000 and 2019. In TNM staging system, stage IB1, IB2, IB3, IIA1 and IIA2 disease were found in $32(31.4 \%), 50(49 \%), 12(11.8 \%), 6(5.9 \%)$ and 2 patients $(2 \%)$, whereas $48(47.1 \%)$ showed different stage by tumor size measured by MRI. In terms of pathologic tumor size $\geq 4 \mathrm{~cm}$, MRI had sensitivity of $30.8 \%$ (4/9), specificity of 95.5\% (85/89), positive predictive value (PPV) of $50 \%(4 / 4)$ and negative predictive value of $90.4 \%$ (85/94), showing accuracy of $91.1 \%$. In regard to the need of adjuvant radiotherapy, MRI showed sensitivity of $40 \%(2 / 5)$, specificity of 96.9\% (94/97), positive predictive value (PPV) of 40\% (2/5) and negative predictive value (NPV) of $96.9 \%$ (94/97), showing accuracy of $93.1 \%$.

Conclusions The accuracy of MRI for predicting TMN stage may not be effective. However, MRI may have high specificity and NPV for preoperative prediction of pathologic tumor size $\geq 4 \mathrm{~cm}$ and the need of adjuvant radiotherapy in early-stage cervical cancer. 